\title{
Dilemmas and Paradoxes in Linguistic Fieldwork
}

\author{
JØRGEN RISCHEL
}

University of Copenhagen, Denmark

\section{Introduction}

Linguistic fieldwork has a long and glorious past in the United States, first and foremost because of the devotion of Boas, Kroeber, Sapir and their followers to the documentation of the numerous native languages of North America. I feel very humble in this context, and it is a great honor for me to have been invited by the Berkeley Linguistics Society to address this topic.

Linguistic fieldwork involves three essential components: native speakers, the target language, and the technical outfit and skills of the field worker. This can be observed, with large variations in emphasis, in the traditional readings of the sixties such as Healey (1964), Longacre (1964), and Samarin (1967), or in the brief statements in Gudschinsky (1967: 4-7) about "Field Procedures" and "Use of Informants." Issues to do with native speakers as individuals and as a speech community are, however, much more in focus nowadays, and personally I feel they should be given the highest priority. The present paper is very broad in scope and may seem almost redundant since there has been an recent upsurge of interest in fieldwork methodology, as witnessed by publications such as Vaux \& Cooper (1999), Newman and Ratliff (2001). Still, I hope to raise some live issues.

There is a general prevalence in linguistic fieldwork of settings involving more or less fluently bilingual speakers. In this presentation, on the other hand, my focus is on the interaction with speakers of oral languages who are monolingual or have at most a very restricted competence in other languages, who are absolutely non-literate (in any language), and who have had limited exposure (or virtually no exposure) to non-native behaviour such as the ways in which they may be approached by field workers. They are the prototype of so-called "unsophisticated informants" (Healey 1964). My reason for this particular focus is that I happen to have been working in such speech communities over the last twenty years.

There used to be one school which explicitly emphasized the necessity of formalizing discovery procedures, namely post-Bloomfieldian structuralism, but in fact its methodology did not specify how fieldwork was to be performed in practice, with the more Sapir-inspired Pike-Nida trend as a notable exception (Nida 1946). 
The main point, as I see it, was a warning against involving native speakers in metalinguistic discussions about the meanings of linguistic forms.

My own experience is that it is difficult and hardly fruitful to steer clear of discussions of meaning. Native speakers are often little interested in whether linguistic tokens are alike or different whereas they are extremely interested in helping the ignorant linguist to understand what an expression means. After all, that is what language is about. When working on a little explored language it is a source of potential error that the field worker and the native speaker misunderstand each other's intentions. The native speaker expects to be engaged in meaningful communication in which the spoken language is the medium rather than the object of study. If eventually it becomes clear that the field worker wants to focus on the language itself, native speakers who have little or no experience with researchers tend to think in terms of synonymy rather than phonetic similarity. There is a priori little inclination to engage in seemingly pointless comparisons of phonetically related words or utterances. Personally I am often in doubt about responses to questions about similar or different? unless I have specified that I want to know whether things have the same "name" or not.

The retrieval of data was never an issue that loomed large in standard textbooks on linguistics. It remained rather peripheral, even to scholars with a long experience in approaching linguistic data. Introductory textbooks teaching morphological or syntactic analysis are often very bad in this respect: they tend to depict raw data as a set of carefully selected and flawlessly transcribed forms. Thus they create a dangerous illusion in the minds of students unless the teacher provides a counterbalance by explaining and demonstrating how crucially important it is for the whole field of linguistics how the raw material used as input was retrieved in the first place.

\section{Phonetic issues}

I myself have attempted to say in various papers over the years (cf. Rischel 1983, 1987, 1989) that one cannot overestimate the importance of the initial confrontation with phonic data and the initial generalizations over data made in the field. Errors of perception or sloppiness in transcription may cast a long shadow over all the ensuing work at home, and even worse: over the work of other linguists who trust the information. Communication breakdown between field worker and native speaker is a more tangible source of error, and the field worker may not detect such breakdowns. There is an additional danger in that one's data may be skewed in some way from the start because of the conditions under which one works. I shall discuss some of these sources of error in the present paper.

One of the most urgent questions in fieldwork is how to achieve phonetic adequacy. I wish to highlight one issue, namely the status of field notes versus electronic recordings. First some words about the choice of electronic recording media: I have found it increasingly rewarding to use video along with or even instead of audio tapes. Especially if one is recording narratives, it is a matter of so-called 


\section{Dilemmas and Paradoxes in Fieldwork}

total communication involving significant mimicry and gestures as, for example, when the narrator uses different points in space deictically to refer to the actants in the story, or uses his hands to show trajectories of movements which are significant in the story. This is an essential part of the encoding of its contents, and audio recordings of narratives may be defective or at least much harder to understand without such visual support.

The importance of video recording is a reflection of the accessibility, in the field situation, of other important sources of information than the sound of the speech itself. Pike spoke of phonemics as a way of reducing speech to writing. I would say that tape recording is in itself a way of reducing oral language: one peels a lot of information off, and this reductionism is of course carried a very big step further if one transcribes the data linearly by leaving out such paralinguistic features as expressive voice quality and variable speech tempo. These phenomena are communicatively crucial and play a paramount role in daily conversation, but unfortunately they do not fit easily into the format of conventional phonetic transcription, and as linguists we have generally not been taught to pay much notice to them. Still, it is easy to point to instances where paralinguistic or extralinguistic phenomena such as voice quality or the direction of the speaker's glances are cues to actants in the story like pronouns, for example.

This paper is not about instrumental phonetic field techniques. Still, I wish to mention that although the access to instrumental analysis either in the field or at home is becoming increasingly rewarding for the purpose of analysis and important for the purpose of documentation, there is an inherent dilemma in modern phonetic fieldwork. It is part and parcel of the professional phonetician's education that much instrumental phonetic analysis requires averaging over several tokens recorded under so similar conditions that it makes sense to process them statistically. In the field, on the contrary, it may be well nigh impossible to monitor the variability; everything changes all the time. What one can hope to get out of a natural field situation, then, is a selection of typical tokens rather than average tokens.

Even as a phonetician I would claim that the most indispensable accessories in the early field situation are not electronic devices but ears, eyes, a pencil and a notebook with good quality paper. I have always found that the notes made in the field have a first-rank status as data, no matter how much electronic recording one has made. Listening in the field and listening to a tape at home are two very different things. In the field one may hear the same word repeated several times and form an impression which integrates the variability occurring over the various tokens. One can listen at a distance and observe the movements of the lips and the jaw at the same time, or one can have one ear close to the speaker's mouth to hear such things as the degree of voicing in stops more clearly. One can then combine these impressions into a faithful and meaningful transcription. On the other hand, one cannot manage to write speech down in real time with the use of phonetic notation, so if the speaker volunteers a lot of data spontaneously but is reluctant or unable to repeat it later, one is at a loss without electronic recording. Especially in the case of narratives I have 
had to rely very much on listening to tapes or viewing videos at home in order to make raw transcriptions which can then be rechecked in the field (hopefully). The electronic recording is also indispensable as a means of lasting scientific documentation, but according to my experience tapes are of very little use to posterity if the field worker does not manage to supply competent transcriptions before handing them over to others.

I shall now switch to a related issue: how to combine accuracy and consistency in the narrow or broad phonetic notation. I have written a paper about this issue several years ago, and I shall just recapitulate a couple of points and add a new comment. As a retired phonetician I ought not to say anything negative about the IPA notation, but I cannot help stating that I think it has inherent problems which appear in fieldwork. One problem is the decision whether to choose a fairly narrow or a fairly broad notation. It makes sense to stick to a very narrow transcription if one makes sure that the phonemic status of each wordform is under control; in that way one captures all the phonetic variation occurring over the data, but a major difficulty is the faithful notation of vowel qualities. To devote a lot of attention all the time to the proper use of diacritics on vowel symbols, may be cumbersome and time consuming in the sometimes stressful field situation. It may even become an unnecessary source of distraction from the issue one is concerned with, especially if the words in question have already been carefully phonemicized and entered into the lexicon.

I have felt this problem particularly when I was taking down phrases or sentences for the purpose of grammatical analysis. If new words occur in the process, I of course transcribe them in narrow IPA, but I have found that for the words that occur all the time I tend to jump immediately to my phonemic image of them which means skipping to a broad transcription so that the overall notation becomes a mixture of narrow and broad transcriptions.

Using a hybrid transcription is all right if one can keep track of the two modes of notation, but there is a serious source of error here because of the nature of the IPA. In this phonetic alphabet a vowel symbol without diacritics has two quite different roles: if used in broad notation it can function as a cover symbol for a variety of vowel qualities within a certain range; in narrow transcription, on the other hand, it denotes a fairly specific vowel quality by virtue of the cardinal vowel convention. If I enter a word from my field notes into my lexicon I must all the time recheck whether vowel symbols without diacritics are meant in the broad or the narrow sense. The problem is particularly serious in the range of open vowels: I honestly think there are too few $a$-symbols in the IPA alphabet. My private solution now is to switch between a fairly narrow IPA and a romanization which I cannot possibly mistake for IPA, and to use the IPA whenever it is relevant in order to capture unpredictable phonetic detail.

\section{Inadequate coverage of linguistic usage}

The data at one's disposal may be incomplete or skewed in numerous ways, and that may have dire consequences at all levels of linguistic analysis and documentation. 


\section{Dilemmas and Paradoxes in Fieldwork}

That is a particularly serious issue if one is working with a language which is moribund or "terminal" (Dorian 1986), so that there may be little chance of repairing inadequacies later. Since it is a vast and confusing topic, I must limit myself to a few types of significant skewedness or non-representativity:

One problem is the choice of speaker. The members of any speech community, even a very small tribe, may be as different as people in the street: some are eloquent, some say less but think more conscientiously about the information they give; others again refuse to perform in a fieldwork setting, because they consider themselves dumb or rustic or because they find (with some justification) that the whole set up is phony. But these same people may thaw up if one manages to get involved in genuine communication about real issues. The most willing and eloquent speakers, or the ones with the best voices, are certainly not always the ones that yield the most genuine information about the language one is studying. I myself once recorded quite a bit of word elicitation with a young boy because he had a clear voice and could control himself so as to speak at a moderate speed, but eventually the material must be scrapped because it turned out that he imitated my own speaking style (with all of its speech errors) out of veneration for me, whom he obviously regarded as a close senior relative. Then again some speakers may speak with a more or less slurred articulation because of missing teeth or other impairments, but these (typically old) people may be a gold mine of information e.g. about lexicon or conservative morphology if one can find out what they are saying. Some of my best field sessions were made with people sitting at leisure, e.g. with a pipe between the teeth. This sometimes brought me-as a phonetician - into despair, but it was a dilemma since attempts to force the speaker to abandon his or her pipe might ruin the mood which was a prerequisite to the volunteering of precious information. There is often a conflict between what satisfies the phonetician and what satisfies the syntactician or the cultural researcher. This is an unfortunate dilemma if the field worker performs in several roles at the same time.

A related challenge is genuine variation across the language community. Although it is convenient to work with just one native speaker and define one's goal as the description of an idiolect rather than a dialect or language in its totality, the speaker may have very idiosyncratic features of phonology and even of grammar or lexicon. Others in the group may speak differently.

I have met with impressive lexical variation in an oral language in Southeast Asia which I am working with: the Austroasiatic hunter-gatherer language Mlabri. It is obviously highly relevant in such cases to know as much as possible about each speaker's family and experiences in life. Some such inter-speaker variation may persist for a long time as idiosyncratic characteristics of individuals or families. In Mlabri this is true of certain strange phonological fluctuations which I observed in a language variety spoken by a tiny and fairly isolated group of twenty-odd persons living in the forest close to the wild border between Northern Thailand and Laos. One of their variational features is an idiosyncratic preference for either a final dental or a final velar stop in the definite article, another is the idiosyncratic substitution of 
a palatal glide for an alveolar lateral. If one looks at the only early recording of the language: an otherwise rather inadequate word list from 1938, it most remarkably shows these very fluctuations. Other inter-speaker differences are more important for an overall characterization of the language since they are associated with socially defined groups; it is noteworthy in particular that a language such as Mlabri exhibits several lexical doublets such that one word of a synonym pair is dubbed as male speech, the other as female speech.

More or less orthogonally to the dimensions of sociolectal and idiolectal variation there is in probably every speech community a variation over speaking styles. This is a challenge to fieldwork. In order to understand the phonological mechanisms of a language it is indispensable to have access to several renderings of the same forms in distinct, normal, and casual style, or whatever style distinctions seem appropriate to make. This is a very important property of language, and it is exploited communicatively. For diachronic-comparative purposes highly distinct forms are often of crucial importance, but speakers may construe artificial pronunciations or make spurious etymological associations if asked to speak over distinctly.

Narratives give access to natural connected speech, to genuine syntax, and to a rich lexicon, but how do we approach narratives in a hitherto unexplored language? And how do we find the ideal speaker? I have always looked with envy at those classic editions of folklore which look as if everything worked smoothly, as if everything was delivered in dictation style by story tellers or by those reciting ritual texts or traditional songs. Like many others $I$ have been recording narratives quite extensively, and that works fine in the recording phase, but I think we all have the experience that the interpretation requires much more extensive fieldwork with the same speaker or with other speakers afterwards. In the case of Mlabri my best material stems from a speaker who is a narrative genius but is half deaf and has now also developed a disease so that he has difficulty phonating. To make things worse he speaks incredibly fast.

The approach I have chosen is to play the recordings back several times to his children who can perceive almost all he says. Their task is to repeat the words exactly so that I get a supplementary, distinct tape recording. The session is monitored by the father who endorses or corrects their rendering. That may sound easy but it did not work smoothly from the start. These youngsters were convinced that I only wanted the contents of each story and that my only problem was lexical incompetence. So they kept paraphrasing the paragraphs I was supposed not to understand, instead of repeating the words more distinctly. As they obviously know how to speak to a half deaf person, my strategy has been to tell them that I myself am handicapped by poor hearing but I treasure their father's exact words. Eventually that helped. Still, all paraphrasing is of value. In fact my Mlabri narrator himself exhibits intriguing verbal variation if he tells a story a second or third time.

To avoid too much skewedness in the representation of speaking styles and of genres I have always found it indispensable to record a lot of small talk beside 


\section{Dilemmas and Paradoxes in Fieldwork}

narratives and lexical material. One way to get the small talk is to work monolingually, as a kind of participant observer. In this way one also avoids interference from another language, but then the problem arises that many native speakers change their way of speaking when speaking to outsiders. I shall return to this issue presently.

\section{The dangers of questionnaires and of interpreters}

A quite different kind of skewedness or downright distortion in the data stems from the field worker asking leading questions to the native speaker or-in a bilingual setting-asking him or her to translate words or sentences from a questionnaire into the native language. The use of a standard questionnaire containing nouns and verbs and phrases which "one can be pretty sure to find everywhere" as a first approach has a long tradition, and it was endorsed by the publication of a field questionnaire in the early years of the CIPL (Cohen 1931).

The success of such an approach certainly depends on the setting. My own experience with forced translation of sentences from a peer language into a huntergatherer language is dismal. I am not happy to make theoretical morphosyntactic points that way. If, for instance, a bilingual speaker is to translate from a peer language with extensive use of particles to the native language which perhaps has lesser use of such particles in certain syntactic structures, the speaker may feel a pressure to come up with equally explicit constructions in his or her mother tongue and thus to produce calques with an atypical or even spurious use of particles. Each language has its typical and more or less unique profile, and if one looks at natural speech it is likely to exhibit a repertoire of truly elegant ways of encoding messages, though these may well be poor in quantifiers, or comparison, or relative constructions, or whatever else the general linguist wants to cover in his or her syntactic elicitation.

In this respect I see no principled difference between heavily morphological languages such as Eskimo and almost isolating languages such as Mlabri, just to mention two languages of traditional hunter cultures with which I myself happen to be familiar. I belong to those who find it more rewarding to attempt to understand the ethos of the language rather than search for reflexes of alleged syntactic universals. The hope, then, is that the non-squinting approach may ensure solid data to be used in typological generalizations.

To be blunt, I never believed in questionnaires at all, and when it comes to the languages of peoples who were traditionally hunter-gatherers such as the Eskimos and the forest people in Southeast Asia, I have found the results of this approach to be sometimes slightly grotesque because of the tendency to cultural bias. One can be sure that the resulting lexical data form no representative sample of genuine, traditional vocabulary. In addition, phrasal data obtained via a questionnaire may not permit valid syntactic generalizations.

Item-per-item elicitation of data from a list may nevertheless be a meaningful first aid, especially if done by a competent linguist. The real danger is that the use of 
questionnaires invites a brute force approach in which minority languages are screened, as it were, by research assistants with little training in linguistic documentation and possibly with no experience in interacting with persons of a quite alien group or in monitoring the performance of hired interpreters.

In what I have already said, I have made some reference to the linguistic vehicle of communication between field worker and native speaker. This leads over to my next issue: how to ensure that interesting information is efficiently transferred without too much intermittent communication breakdown.

I want to say a couple of bad things about the use of interpreters. Firstly, an interpreter may, like any person with real or apparent authority, intimidate the native speaker and compromise the genuineness of the data. A field worker performing as a half proficient participant observer stands a better chance of not having this influence (but then he or she may influence the native speaker's language use in other ways; see above and below). Secondly, if the interpreter and the native speaker communicate via a common lingua franca which the fieldworker may not master, there are sources of error at all stages when a question is transmitted successively from the field worker's language via the interpreter's use of the lingua franca to the native speaker who attempts to understand what the interpreter said, or when the answer is transmitted back.

\section{Monolingual fieldwork}

The very opposite procedure of those mentioned so far is to work directly with a native speaker, without any interpreter and perhaps even with no shared language of communication, especially if the target language is little known or hitherto unexplored and is spoken by a relatively isolated group. Most people probably start such a session by showing objects or actions, directly or via pictures. One can indeed get a basic vocabulary that way (although pictures easily confuse people who are not accustomed to visual representations of things, cf. below). But eventually the range of easily accessible items to be presented and identified is exhausted, and I know by personal experience that this may lure the field worker into falsely believing that the field notes now give a fair coverage of the lexicon. After all this is a tribal language! The truth, of course, is that so-called "small" languages are extremely rich and complex when it comes to semantic fields which are culturally or socially important.

I greatly prefer a setting in which one can communicate monolingually with the native speaker (also cf. Everett 2001). It is true that this approach may be frustrating if one does not understand enough of what is said, so that a lot of information is lost. Also misunderstandings may arise e.g. if the field worker's questions are too poorly phrased.

On the other hand native speakers are both highly motivated and free of anxiety so they are at leisure to explain carefully and instructively provided that the main vehicle of communication is their own language. This means that whatever the field worker really understands tend to be fairly reliable. Practically all I know about the rich Mlabri vocabulary of verbs that refer to body postures and body movements was 


\section{Dilemmas and Paradoxes in Fieldwork}

communicated to me monolingually and explained visually. It did not take too much speaking proficiency to ask simple questions such as: "If you want me to sit like this, or to move my hand like this, how then do you say that?"

In the case of nouns, on the other hand, the lack of a fluent reference language may create difficulties with the retrieval of lexical information as soon as one proceeds beyond objects within easy reach. As for animals (including insects and worms) and plants in the forest, I have had disappointingly little success in attempting to cover the native Mlabri lexicon adequately.

Even showing charts of different species may create confusion with "unsophisticated" native speakers if consecutive pictures are not to the same absolute scale. I have been using a picture book with local birds in which each colour chart typically included two or more different species as if they lived close together, and then a different problem arose: birds in such a chart were often identified as tokens of the same species, e.g. as 'fatherbird', 'motherbird' and 'offspring'. It is also my experience that some of those consulted take pride in identifying as many species as possible, at the expense of accuracy, whereas others takes the opposite stand and hardly make any wholehearted identifications. It goes without saying that there are much more professional approaches to such elicitation by means of photographs, but in the field situation, especially inside a mountain forest, it tends to be a matter of what is practical.

For many lexical entries I just have more or less vague descriptions or sometimes broken, incomplete or decaying parts. I have had to consider it impractical to make extensive zoological and botanical safaris together with experts in the natural sciences in order to find the objects in situ, both because the presence of several outsiders easily ruins the hard-won, relaxed atmosphere of monolingual fieldwork with shy native speakers, and also because of practical problems (the Mlabri suggest that some information retrieval is best accomplished by climbing trees, which even to them themselves is sometimes dangerous). Therefore, many such entries in my notebooks have pseudogeneric, descriptive translations like 'species of tree described as tall, with small leaves and inedible fruits, growing high up in the forest' or 'inedible tuber species' or '(presumably:) cicada species, not eaten'. This is not satisfactory, but I think it is potentially more misleading for users of the data if one makes a posteriori identifications and supplies a Latin name for such a species on shaky evidence. The most important thing is to strive not to supply ill-founded information on the basis of guesswork.

The alpha and omega of lexical fieldwork is to recheck one's data over and over again in different situational contexts or at least in different discourse contexts. But that may be difficult when it comes to words for things which are outside one's own range of experience. As for animals and plants in the deep forest the Mlabri sometimes volunteer a Thai or Lao name of such an animal or tree, but their use of Thai or Lao is not reliable. I once recorded a mythological narrative of The-GreatFlood-Myth type, and when I went over it with the story teller he explained about a white bird flying over the ground after the natural disaster with fresh seeds in its 
crop. I was in doubt about the Mlabri word for the bird, and he then explained in the local lingua franca that it was an owl. This sounded spurious, so I later showed him pictures of an owl and a pigeon. Unsurprisingly, he pointed at the pigeon but said in the lingua franca: "Yes, it was an owl!"

We all know that one of the best ways of getting genuine everyday speech is to spend time on engaging in conversations about relevant topics. In this way one also stands a much better chance of recording vocabulary with correct glossing. The ideal case is for a situation to arise which invites such a conversation. Once, for example, a young Mlabri woman suddenly felt an urge to explain in very much detail about her recent troubles giving childbirth. The child's grandmother afterwards gave her version of the whole event, and others present elaborated on the story so as to make sure that I had understood every part of this drama. The focus was not at all on the language but on the components of such an event, but since they were eager to make me understand, I got a lot of new vocabulary, and it became an exemplaric case of interactive linguistic fieldwork.

Still, a monolingual approach has its shortcomings. The output may be biased by the nature of the communication. This problem is shared with sociolinguists of the Labov brand making a so-called "sociolinguistic interview." One should be prepared for a shortage of reliable data on interrogative constructions, for example, if one uses an interactive approach. There are several reasons for that. Firstly, the situation itself may not invite a lot of real questions put $b y$ the native speaker to the field linguist. In addition, it may be awkward for the tribesperson to address a quest for information to a seemingly more prestigious outsider. The field worker may then attempt to provoke samples of question types, but the sensible native speaker is likely to find it weird to be asked to produce a pointless question. It is obviously preferable for the linguist to stay for a long time in the community and to pick up questions from conversations between tribespeople. It should not be overlooked, however, that in-depth processing of such material actually requires very thorough familiarity not only with the language as such but with the whole communicative context and with the communicative strategies used in the speech community, with expected presuppositions and with speech act types. It may be easier and in the initial phase more rewarding to look at data for which the contextual setting is much simpler than in natural conversation. I have found it useful to look at questions imbedded in folk tales in which the characters talk together. If at times one does not understand the reason why this or that question occurs in the tale, one can be sure that the native speakers will gladly volunteer to explain that in accordance with their interpretation of the narrative.

A further danger in the monolingual setting is that the unfortunate field worker may happen to be retrieving spurious data on connected speech. I mentioned earlier that native speakers may choose, perhaps inadvertently, to adjust or even distort their speech so as to accomodate to the field worker. Such distortion, particularly in the form of excessive simplification in morphosyntax and lexicon, may also occur if the native speaker detects that the outsider's linguistic proficiency is poor. Predecessors 
may cast a shadow of this kind. One elderly couple in Northern West Greenland spoke to me in an almost unintelligible kind of pidgin Greenlandic, largely without inflections, which is serious in a polysynthetic language. The reason turned out to be that they had been hired for practical work by the local Danish school teacher who had arrived several years before with little command of the language, so they had chosen that strategy rather than confuse him with inflections. This was an easily detectable pidgin situation, but in less extreme cases the field worker may remain for a while in happy ignorance about the spuriousness of the linguistic input, especially if it is his or her first encounter with a little-known language. Hopefully, the uncomfortable truth reveals itself sooner or later.

\section{The ethics of fieldwork}

I wish to round off by emphasizing that some of the most serious dilemmas have to do with the basic ethics of fieldwork. Speakers are not automata but members of a speech community. We must all the time ask ourselves: do we understand their attitude, and do we know what is the appropriate way of showing our appreciation of their help? Some native speakers offer their help freely and without limits if they feel that the endeavour may assist the survival or at least the memory of their language and culture. They may react very differently if they assume that the purpose of the fieldwork is to gain a personal advantage on the basis of shallow knowledge. Sometimes the quality of our field data depends on the extent to which the native speaker can see the point in painstaking accumulation of seemingly trivial information.

In the field we are forced to acknowledge that a language is not just an autonomous object but the backbone of the cultural make up of its speakers. Access to a language also means access to facets of spiritual culture which would otherwise remain inaccessible. But the retrieval of information always comes at a cost. In one sense we serve the cause of humanity by struggling to document a vanishing language and culture, but in doing so we may violate the integrity of its bearers if we ask persistent and seemingly silly questions about matters which to them are sensitive. Researchers in the field must take a stand on such issues personally, and I hope we all do.

\section{References}

Cohen, Marcel. 1931. Questionnaire Linguistique. Publications de la Commission d'Enquète Linguistique. Comité International Permanent de Linguistes.

Dorian, Nancy C. 1986. Gathering language data in terminal speech communities. In Jushua A. Fishman et al. (eds.) The Fergusonian Impact. In Honor of Charles A. Ferguson. Vol. 2: Sociolinguistics and the Sociology of Language, 555-75.

Everett, Daniel L. 2001. Monolingual field research. In Newman, Paul \& Martha Ratliff (eds.) Linguistic Fieldwork, 166-88. 
Gudschinsky, Sarah C. 1967. How to Learn an Unwritten Language. New York: Holt, Rinehart and Winston.

Healey, Alan. 1964. Handling Unsophisticated Linguistic Informants. Linguistic

Circle of Canberra Publications, Series A: Occasional Papers 2. Canberra: Linguistic Circle of Canberra.

Longacre, Robert E. 1964. Grammar Discovery Procedures: A Field Manual. The Hague: Mouton \& Co.

Newman, Paul \& Martha Ratliff (eds.). 2001. Linguistic Fieldwork. Cambridge: Cambridge University Press.

Nida, Eugene. 1946. Field techniques in descriptive linguistics. International Journal of American Linguistics 13.3: 138-46.

Rischel, Jørgen. 1983. Fieldwork data as input to instrumental analysis: A dilemma. In Pieper, Ursula \& Gerhard Stickel (eds.) Studia Linguistica Diachronica et Synchronica, 709-22. Berlin: Mouton de Gruyter.

Rischel, Jørgen. 1987. Phonetic transcription in fieldwork. In Almeida, António \& Angelika Braun (eds.) Probleme der phonetischen Transskription. Zeitschrift für Dialektologie und Linguistik, Beiheft 54, 57-77. Stuttgart: Franz Steiner Verlag.

Rischel, Jørgen. 1989. Fifty years of research on the Mlabri language. A re-appraisal of old and recent fieldwork data. Acta Orientalia 50: 49-78.

Samarin, William J. 1967. Field Linguistics. New York: Holt, Rinehart and Winston. Vaux, Bert \& Justin Cooper. 1999. Introduction to Linguistic Field Methods. München, Newcastle: Lincom Europa.

Stenhøjgaardsvej 57

DK-3460 Birkerød

Denmark

jr@cphling.dk 